THE AstrophysicAL JoURNAL, 544:937-943, 2000 December 1

(c) 2000. The American Astronomical Society. All rights reserved. Printed in U.S.A.

\title{
STELLAR COMPANIONS AND THE AGE OF HD 141569 AND ITS CIRCUMSTELLAR DISK
}

\author{
A. J. Weinberger, ${ }^{1}$ R. M. Rich, ${ }^{1}$ E. E. Becklin, ${ }^{1}$ B. Zuckerman, ${ }^{1}$ ANd K. Matthews ${ }^{2}$ \\ Received 2000 March 30; accepted 2000 July 13
}

\begin{abstract}
We investigate the stellar environment of the $\beta$ Pictoris-like star HD 141569 with optical spectroscopy and near-infrared imaging. The B9.5 Ve primary and two other stars (types M2 and M4) both located in projection less than $9^{\prime \prime}$ away have the same radial velocity and proper motion and therefore almost certainly form a triple star system. From their X-ray flux, lithium absorption, and location on pre-mainsequence evolutionary tracks, the companions appear to be $5 \mathrm{Myr}$ old. HD 141569A is now one of the few main-sequence stars with a circumstellar disk that has a well-determined age. The circumstellar disk is composed of secondary debris material, thus placing an upper limit on the time needed for disk processing. These three stars may be part of an association of young stars.
\end{abstract}

Subject headings: binaries: visual — circumstellar matter — stars: individual (HD 141569) — stars: late-type

\section{INTRODUCTION}

Stars with far-infrared excesses generated in circumstellar disks were discovered in abundance by the IRAS mission. Since circumstellar material disappears over time due to processes such as stellar winds, radiation pressure, and accretion onto stars and planetesimals, most infrared excess stars are young, $\lesssim 10^{8}$ yr (Habing et al. 1999; Spangler et al. 1999; Silverstone 2000). To develop an understanding of the evolution of circumstellar material and its relationship to the formation of planets, detailed studies of individual disks must be combined with information about stellar ages. However, stars which are nearing the main sequence are difficult to age date because they lack traditional indicators such as lithium and X-ray emission and they move through the color-magnitude diagram very quickly.

The Herbig AeBe stars (HAEBEs) are thought to be in this transition phase, intermediate in evolution between protostars and stars on the zero-age main sequence (ZAMS). The classical definition of HAEBEs, i.e., that they are of spectral type A or earlier, are found in clouds, and show emission lines and reflection nebulae (Herbig 1960), almost certainly selects for such young objects. In addition, more recent studies (The, de Winter, \& Perez 1994) use the presence of near- or far-infrared excess to select HAEBEs. Thus, objects such as HD 141569, a B9.5 Ve star with $\mathrm{H} \alpha$ in emission and 12-100 $\mu \mathrm{m}$ excess, which are not associated with any cloud or reflection nebula, fall into the HAEBE class. There have been attempts to date HAEBE stars within this general picture based on the strength of their infrared excesses (Hillenbrand et al. 1992), and authors have proposed an evolutionary sequence from the embedded HAEBEs to the isolated $\beta$ Pictoris or Vega-like mainsequence stars (e.g., Malfait, Bogaert, \& Waelkens 1998).

The presence of lower mass companions to these objects represents an independent way of measuring their age, assuming they and the companions are coeval. Lower mass companions may not yet be on the ZAMS, and their ages can be estimated from theoretical pre-main-sequence evo-

\footnotetext{
${ }^{1}$ Department of Physics and Astronomy, University of California, Los Angeles, Box 156205, Los Angeles, CA 90095; alycia@astro.ucla.edu, rmr@astro.ucla.edu, becklin@astro.ucla.edu, ben@astro.ucla.edu.

2 Palomar Observatory, California Institute of Technology, 320-47, Pasadena, CA 91125; kym@caltech.edu.
}

lutionary tracks. For early-type stars near the ZAMS which also have circumstellar dust, this is probably the most accurate way to determine the disk age. The well-studied disk stars, $\beta$ Pic, Fomalhaut, and HR 4796A, were all dated by their association with lower mass comoving companions (Barrado y Navascués et al. 1997, 1999; Stauffer, Hartmann, \& Barrado y Navascués 1995).

HD 141569 is joining the ranks of well-studied dusty disk stars with the discovery of its large and morphologically complicated debris disk (Silverstone et al. 1998; Weinberger et al. 1999; Augereau et al. 1999; Fisher et al. 2000). In 1938, Rossiter identified HD 141569 as a member of a potential triple system with a second star (B) 7.5 away and a third star (C) 1".5 from it (Rossiter 1943). In a study of HAEBEs, Gahm, Ahlin, \& Lindroos (1983) measured the spectral type of HD 141569B as G0 V, and Lindroos (1985) concluded, based on its magnitude and color, that it was a background star. However, Gahm et al. (1983) noted the presence of "peculiar" emission lines in the spectrum of HD 141569B, including $\mathrm{Ca} \mathrm{H}$ and $\mathrm{K}$ and $\mathrm{H} \beta$, which are often associated with young stars.

The parallactic distance to HD 141569A was measured by the Hipparcos mission as $99 \pm 10 \mathrm{pc}$, which, combined with its visual magnitude of 6.8 , makes it underluminous for its spectral type, just as are other young A-type infrared excess stars such as $\beta$ Pic, 49 Ceti, and HR 4796A (Jura et al. 1998; Lowrance et al. 2000) (see also Fig. 6).

Given the apparent proximity of two stars to HD 141569A, and given that the disk around HD 141569A extends to half of the projected distance between HD $141569 \mathrm{~A}$ and $\mathrm{B}$, this system could be dynamically interesting if the three stars are physically associated. In this paper we show, via comparison to previous astrometry plus new near-infrared imaging and visual spectroscopy, that HD 141569 A/B/C form a triple system, and we estimate their ages.

\section{OBSERVATIONS AND DATA ANALYSIS}

\subsection{HST and Ground-based Imaging}

On 1998 September 27, short integration time images of the HD 141569 system were obtained with the NICMOS Camera 2 on the Hubble Space Telescope in order to acquire the primary star for coronagraphic observations. Two simple ACCUM mode images of $0.342 \mathrm{~s}$ each were taken 


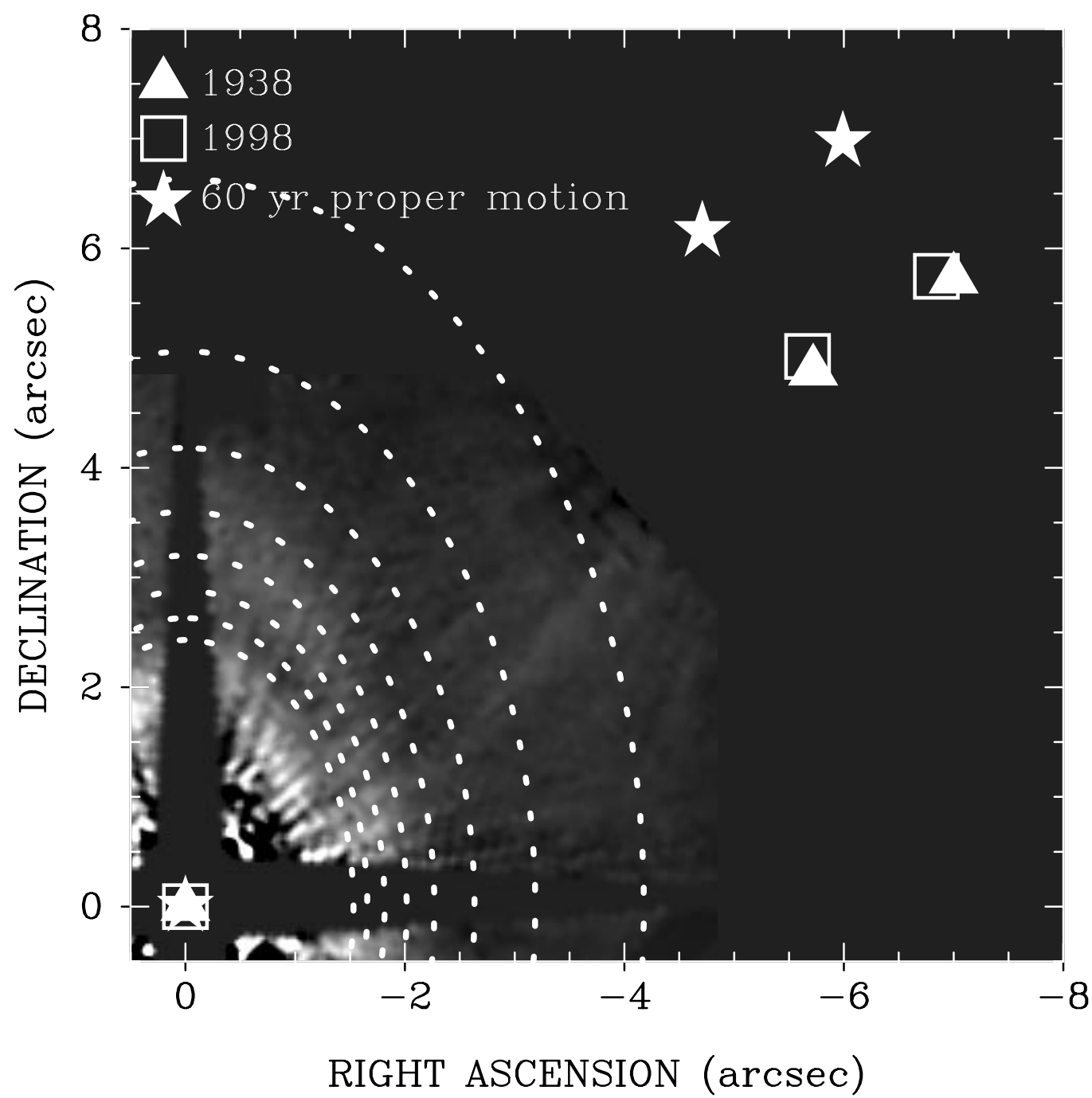

Fig. 1.-Positions of stars B and C relative to HD 141569A shown for two epochs: 1938 (filled triangles) and 1998 (or 1999; open squares). Also shown are the predicted positions of B and C, if they are stationary background stars, for 1998 given their 1938 locations and the proper motion of A over the last 60 years ( filled stars). The sizes of the points are larger than the uncertainties reported in Table 1 in each case. The agreement between the 1938 and 1998 measurements shows that A, B, and C are a comoving group. A NICMOS image of the disk (Weinberger et al. 1999) is shown overplotted with dashed ellipses that give the locations (from outside in) of the 2:1-9:1 Lindblad resonances between $\mathrm{A}-\mathrm{B} / \mathrm{C}$ and disk orbital periods, assuming $\mathrm{B}$ and $\mathrm{C}$ lie in the plane of the disk. In projection, $1^{\prime \prime}=100 \mathrm{AU}$.

with the F171M filter $\left(\lambda_{\text {eff }}=1.721\right.$, FWHM $\left.=0.071\right)$, and 10 dark frames of the same integration time were taken 2 days earlier for calibration of those acquisition images. The two source images were used for cosmic-ray rejection by taking the minimum of the two values for every pixel. The median of the dark images was subtracted from the science image to remove the effects of detector shading and bias. The resulting image was divided by an on-orbit flat-field frame taken in the same filter. The best available photometric calibration was applied in which $0 \mathrm{mag}=948 \mathrm{Jy}$ and $1 \mathrm{adu} \mathrm{s}^{-1}=1.071 \times 10^{-5} \mathrm{Jy}$.

Near-infrared $J-\left(\lambda_{c}=1.27, \Delta \lambda=0.25\right), H-\left(\lambda_{c}=1.65\right.$, $\Delta \lambda=0.32), \quad K-\quad\left(\lambda_{c}=2.20, \quad \Delta \lambda=0.40\right), \quad$ and $L_{s}$-band $(\lambda=3.45, \Delta \lambda=0.57)$ images were taken with the Hale 200 inch Telescope on 1999 May 25 and 28. The infrared camera had a pixel scale of $0^{\prime \prime} 125$ pixel $^{-1}$ and a full field of view of $32^{\prime \prime} \times 32^{\prime \prime}$. The night of May 25 was cloudy, but the flux ratios of B and $\mathrm{C}$ to star A were measured at $J, H$, and $K$ bands. Short integration time images, $0.374 \mathrm{~s}$, were taken, in which all three stars appear in every full-field frame. The seeing was 0 ".82 at $K$ band, so stars B and C were easily resolved. So as not to saturate HD 141569A at $J$ and $H$ bands, the chopping secondary was used to smear the light in a direction perpendicular to the position angle (P.A.) 301.9 between components $\mathrm{B}$ and $\mathrm{C}$.

The night of May 28 was photometric, and measurements of HD 141569A at all four wavelengths were preceded by measurements of the photometric standard HD 129655. For both target and standard, an 8 " square subframe of the full array was employed to allow fast readout and prevent the images from saturating the bright primary at $J$ and $H$ or the thermal background at $L_{s}$. At each filter, 100 integrations of $0.07 \mathrm{~s}$ were co-added for a total on-source integration time of $7 \mathrm{~s}$. One $0.54 \mathrm{~s}$ image of HD $141569 \mathrm{~B}$ and C was also obtained at $L_{s}$.

Sky frames of the same integration time were obtained after every set of exposures on the stars. After sky subtraction, the images were flat-fielded and corrected for hot pixels. Aperture photometry was then performed on the final images. 


\subsection{Spectroscopy from the W. M. Keck Observatory}

Resolution $\sim 5000$ spectra of stars B and C were taken with the LRIS (Oke et al. 1995) instrument on 1999 February 25 covering the spectral range of $6250-7550 \AA$. A long slit of width 0.7 was placed at a P.A. of 301.9 to obtain simultaneous spectra of both $\mathrm{B}$ and $\mathrm{C}$, and two integrations of $120 \mathrm{~s}$ each were made. The amplifier bias was estimated from the CCD overread areas and subtracted from the spectra. A spectral flat field was made from halogen lamp exposures taken immediately after the spectra and was divided into the spectra. The seeing was 1 ."1, so B and C were not completely spatially resolved; their spectra were deblended by fitting the spatial dimension at every spectral element with two one-dimensional Gaussians plus a linear background. The two observed spectra of each star were then averaged.

High resolution spectra of all three stars were obtained with HIRES (Vogt et al. 1994) on 1999 July 19. The C1 decker was employed, which gives a slit 0 ".86 wide and 7" long, yielding $R=45,000$ over 21 orders, from 5420 to 7880 $\AA$ with some gaps. The small separation of $B$ and $C$ required attention to scattered light. Hence, the image rotator was used to align the slit so that it was perpendicular to the vector separating the stars. Because of the good (0"8) seeing, small slit width, and care in position of the slit, we believe that the individual spectra of $\mathrm{B}$ and $\mathrm{C}$ are uncontaminated by scattered light from the other components. A radial velocity standard, HR $6056\left(-19.9 \mathrm{~km} \mathrm{~s}^{-1}\right)$, was observed immediately following the spectroscopy of HD 141569 $\mathrm{A} / \mathrm{B} / \mathrm{C}$. The spectra were extracted using the MAKEE package written by T. Barlow; however, equivalent widths and radial velocities were measured using the SPLOT package in IRAF.

\section{RESULTS}

\subsection{Relative Motion}

A summary of measurements of relative separation and orientation of HD $141569 \mathrm{~A} / \mathrm{B} / \mathrm{C}$ is given in Table 1 and Figure 1. The first point, from 1938 (Rossiter 1955), averages four measurements of B-C and three measurements of A-B made in 1938 and 1943. Although no uncertainties were given for the measured separations and P.A.s, we used the scatter in the measurements to estimate them. The 1998 data are from our NICMOS acquisition images and the 1999 data from our Palomar images. The uncertainties for the Palomar positions were found from the standard deviation of many independent measurements, as described in $\S$ 3.4. From 1938 to 1998 , the motions of the three stars are negligible to within the uncertainties, and therefore they all have the same proper motion.

In 1995-1996, Pirzkal, Spillar, \& Dyck (1997) imaged HD $141569 \mathrm{~A} / \mathrm{B} / \mathrm{C}$ with shift-and-add and measured the A-B

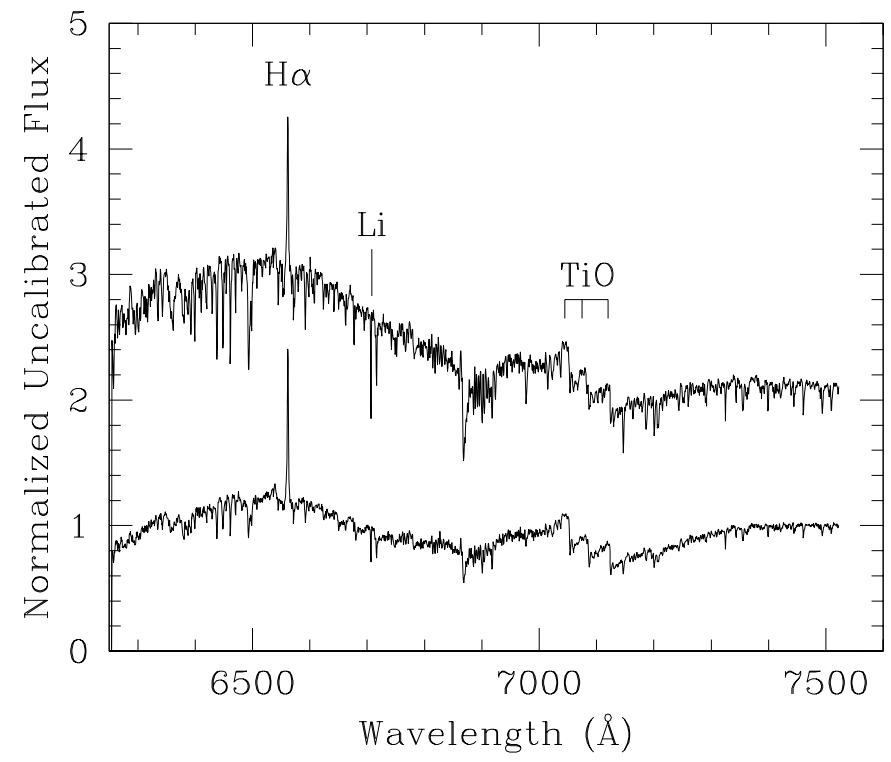

FIG. 2.- Low-resolution spectra of stars B (top) and C (bottom). Units of flux are arbitrary, since the spectra were not flux-calibrated and global slopes are not meaningful. Apparent are $\mathrm{H} \alpha$ in emission and $\mathrm{Li} \lambda 6708$ in absorption, as well as strong $7000 \AA \mathrm{TiO}$ bands.

separation as 6.8 at a position angle of $312^{\circ}$ and $\mathrm{A}-\mathrm{C}$ as 8.0 with P.A. $=314^{\circ}$. No uncertainties were provided in that paper, and these results are inconsistent with the others.

\subsection{Spectral Types and Features}

The LRIS spectra of stars B and C are shown in Figure 2. Notable features are $\mathrm{H} \alpha$ in emission, $\mathrm{Li}$ I $\lambda 6708$ in absorption, and the TiO bands at $\sim 7000 \AA$, which all appear in both spectra. The spectra have not been divided by a spectral standard, so global slopes are not meaningful. Figure 3 presents a portion of the LRIS spectrum of B compared to spectral standard stars in Kirkpatrick, Henry, \& McCarthy (1991). Figure 4 does the same for C. We assign a spectral type of M2 V to B and M4 V to C, based on the depth of the TiO features.

The equivalent widths of $\mathrm{H} \alpha$ and $\mathrm{Li}$ I are presented in Table 2 and the $\mathrm{H} \alpha$ line profiles for $\mathrm{B}$ and $\mathrm{C}$ are shown in Figure 5, both from the HIRES spectra. Stars B and C have double-peaked lines, with nearly the same shape and separations of their peaks of $1 \AA$. $\mathrm{H} \alpha$ in star $\mathrm{A}$ is much broader, double-peaked with a peak-to-peak separation of $5.3 \AA\left(242 \mathrm{~km} \mathrm{~s}^{-1}\right)$ and has a stronger blue than red peak, all of which are consistent with previous measurements of $\mathrm{H} \alpha$ by several authors (Andrillat, Jaschek, \& Jaschek 1990; Zuckerman 1994; Dunkin, Barlow, \& Ryan 1997).

TABLE 1

SUMMARY OF ASTROMETRY

\begin{tabular}{|c|c|c|c|c|c|}
\hline \multirow[b]{2}{*}{ DATE } & \multirow[b]{2}{*}{ REFERENCE } & \multicolumn{2}{|c|}{ A-B } & \multicolumn{2}{|c|}{ B-C } \\
\hline & & $\begin{array}{l}\text { Separation } \\
\quad(\operatorname{arcsec})\end{array}$ & $\begin{array}{l}\text { P.A. } \\
\text { (deg) }\end{array}$ & $\begin{array}{l}\text { Separation } \\
\quad(\operatorname{arcsec})\end{array}$ & $\begin{array}{l}\text { P.A. } \\
\text { (deg) }\end{array}$ \\
\hline $1938 \ldots \ldots$ & Rossiter & $7.5 \pm 0.1$ & $310.5 \pm 0.5$ & $1.5 \pm 0.1$ & $302.7 \pm 0.5$ \\
\hline $1998 \ldots \ldots$ & NICMOS & $7.57 \pm 0.01$ & $311.5 \pm 0.1$ & $1.38 \pm 0.01$ & $301.9 \pm 0.1$ \\
\hline $1999 \ldots \ldots$ & Palomar & $7.55 \pm 0.02$ & $311.4 \pm 0.1$ & $1.37 \pm 0.01$ & $302.0 \pm 0.3$ \\
\hline
\end{tabular}




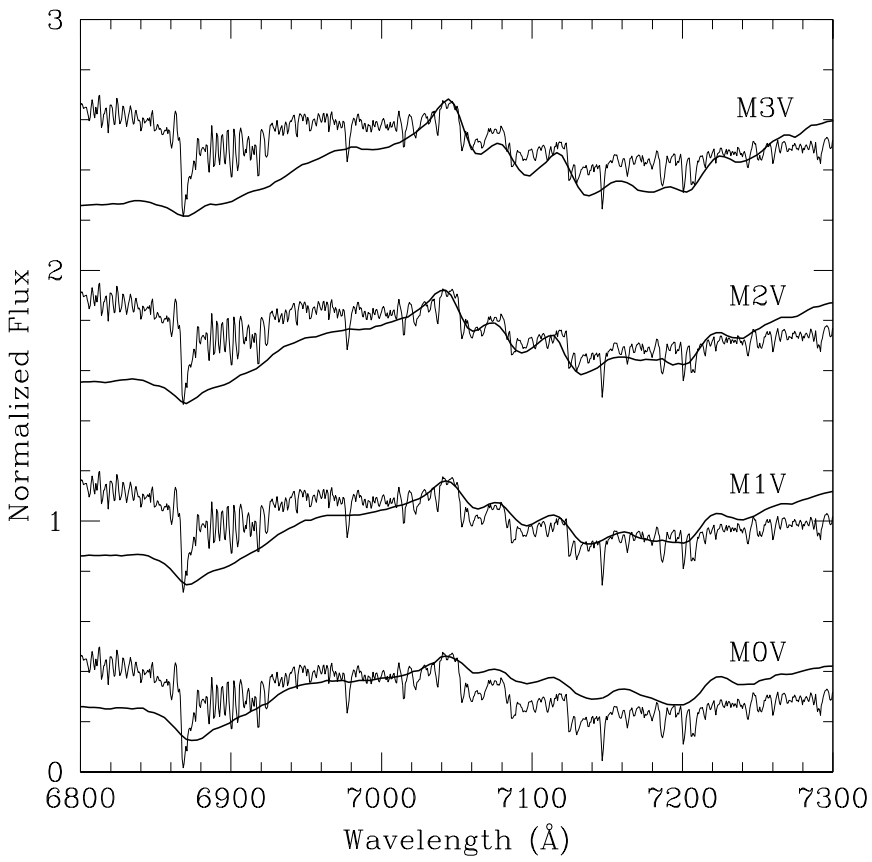

FIG. 3.-Detail of the spectrum of star B centered on the TiO bands at $\sim 7000 \AA$, compared to spectral standards of types M0 V-M3 V from Kirkpatrick et al. (1991). Again, global slopes are not meaningful, since the spectrum was not divided by that of a spectral calibrator. The bestmatched standard is of type M2 V.

\subsection{Radial Velocity}

From a cross-correlation of the high-resolution B and C spectra over each of the 21 spectral orders, B is moving away from us faster than $\mathrm{C}$ by $0.9 \pm 0.4 \mathrm{~km} \mathrm{~s}^{-1}$. The uncertainty is the standard deviation in the mean of the 21 crosscorrelations. The radial velocity of $\mathrm{B}$, from cross-correlation between its spectrum and that of the standard, is

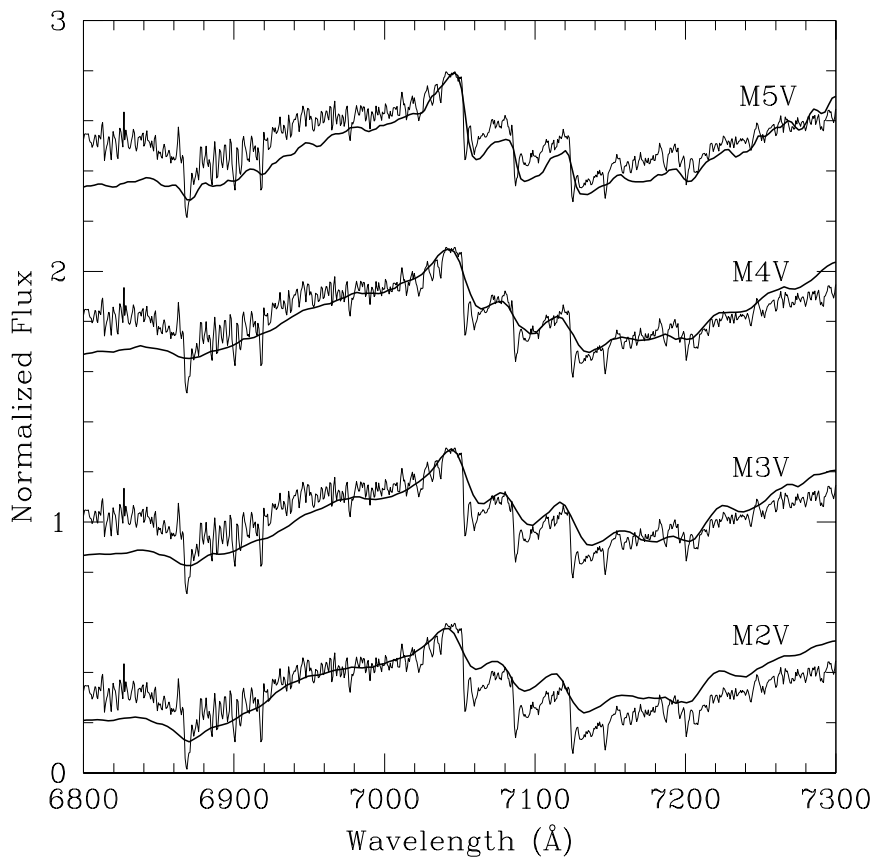

FIG. 4.-Detail of the spectrum of star $\mathrm{C}$ centered on the TiO bands at $\sim 7000 \AA$ A compared, to spectral standards of types M2 V-M5 V from Kirkpatrick et al. (1991). The best-matched standard is of type M4 V.

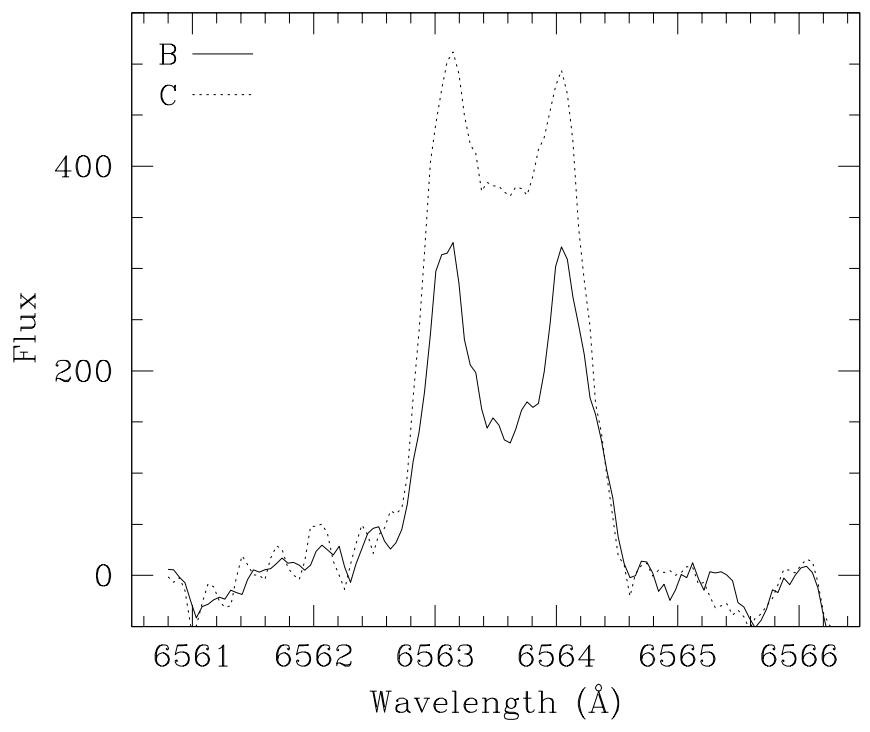

FIG. 5.- $\mathrm{H} \alpha$ line profiles of stars $B$ and $\mathrm{C}$ after continuum subtraction. The double-peaked structure, indicative of chromospheric activity, is of the same width in both stars, although the line equivalent widths and depths of the central reversal differ.

TABLE 2

Equivalent Widths and Heliocentric Velocities

\begin{tabular}{|c|c|c|c|}
\hline \multirow[b]{2}{*}{ STAR } & \multicolumn{2}{|c|}{$\begin{array}{l}\text { EW } \\
(\AA)\end{array}$} & \multirow{2}{*}{$\begin{array}{c}V_{\odot} \\
\left(\mathrm{km} \mathrm{s}^{-1}\right)\end{array}$} \\
\hline & $\mathrm{H} \alpha$ & Li I $\lambda 6708$ & \\
\hline A...... & $-5.51 \pm 0.05$ & & $-6 \pm 5$ \\
\hline B..... & $-0.50 \pm 0.04$ & $0.50 \pm 0.04$ & $-1.3 \pm 1.0$ \\
\hline C $\ldots \ldots$ & $-1.70 \pm 0.05$ & $0.50 \pm 0.04$ & $-2.4 \pm 1.1$ \\
\hline
\end{tabular}

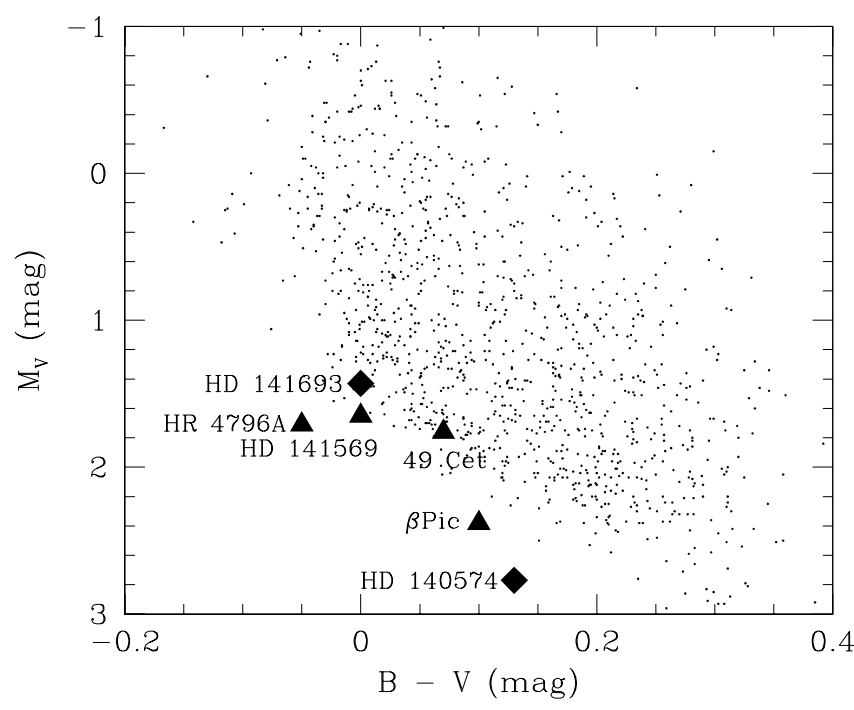

Fig. 6.-All A-type stars (small circles) from the Yale Bright Star Catalog plotted as points on this Hertzprung-Russell diagram reproduced from Jura et al. (1998). Examples of young stars with well-known infrared excesses are plotted with large triangles and fall along the bottom of the distribution. Two A-type stars with the same distance and proper motion as HD 141569, HD 141693, and HD 140574 (large diamonds) also fall in this region of the diagram and, thus, also appear to be young. The absolute magnitudes of the named stars were calculated from their Hipparcos apparent magnitudes and distances and have been dereddened according to their spectral types and $A_{v}=3 E(B-V)$. 
TABLE 3

РнOTOMETRY OF HD 141569 SYSTEM (Vega Magnitudes)

\begin{tabular}{crcccc}
\hline \hline Star & \multicolumn{1}{c}{$J$} & $H$ & F171M & $K_{s}$ & $L_{s}$ \\
\hline A $\ldots \ldots .$. & 6.88 & 6.84 & 6.87 & 6.83 & 6.68 \\
B $\ldots \ldots$. & 9.52 & 8.82 & 8.69 & 8.64 & 8.40 \\
C $\ldots \ldots$. & 10.16 & 9.44 & 9.41 & 9.25 & 8.93 \\
\hline
\end{tabular}

NoTE.-Statistical uncertainties are $4 \%$ at $J, H$, and $K, 2 \%-3 \%$ at $\mathrm{F} 171 \mathrm{M}$, and $5 \%$ at $L_{s}$. No reddening corrections have been applied.

$-1.5 \pm 0.6 \mathrm{~km} \mathrm{~s}^{-1}$. We did not determine the radial velocity of $\mathrm{A}$ because it had no lines suitable for comparison with the M0.5 III spectral-type radial velocity standard. From the literature, its radial velocity is $-6 \pm 5 \mathrm{~km} \mathrm{~s}^{-1}$ (Frisch 1987; Dunkin et al. 1997).

\subsection{Photometry}

In each photometric (i.e., May 28) Palomar image, the magnitude of HD 141569A was measured in a $8^{\prime \prime}$ diameter aperture. In every short nonphotometric (i.e., May 25) frame containing all three stars, A was used as a point-spread function (PSF) for fitting the locations of $B$ and $C$ and flux ratios $\mathrm{B} / \mathrm{A}$ and $\mathrm{C} / \mathrm{A}$, via minimization of the $\chi^{2}$. All 40 images taken at $J$, and 20 images taken in each of the $H$ and $K$ bands, were fit independently. Once the flux ratios were determined, the magnitudes of $\mathrm{B}$ and $\mathrm{C}$ were calculated from the photometry of star A. The results are summarized in Table 3. The uncertainties reported are purely statistical and contain the statistical uncertainty in the magnitude of $\mathrm{A}$ and the standard deviation of the independent determinations of the flux ratios to B and C. In the photometric $L_{s}$ image, the total flux from both $\mathrm{B}$ and $\mathrm{C}$ was measured in an $8^{\prime \prime}$ diameter aperture centered between the stars, and then star B was used as a template PSF to fit the flux ratio of these two close stars.

\section{DISCUSSION}

\subsection{Companionship and Associations}

The proper motion of component A has been measured by Hipparcos as $-16.86 \pm 0.98$ mas $^{\mathrm{yr}^{-1}}$ in right ascension

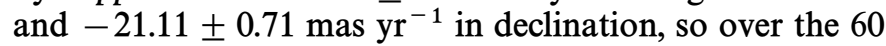
years between Rossiter's measurements and our own, it should have moved 1".01 west and 1".27 south. If B is a background star with negligible proper motion, the P.A. of the A-B pair would have gone from $310^{\circ} .5$ in 1938 to 323.5 in 1998 due to the proper motion of A (Fig. 1). A change of this magnitude is ruled out by the measurements. Furthermore, as can be seen from Table 2, all three stars have consistent radial velocities.

If stars B and $C$ were ordinary main sequence $M$ warfs, their photometric distance would be $\sim 35$ pc. From the Hipparcos catalog, the average proper motion of the 217 stars at this distance, which are within $30^{\circ}$ of HD 141569A,

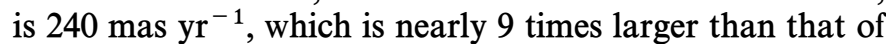
HD 141569A itself. The relative separations of A-B and A-C would have most likely changed by $\sim 15^{\prime \prime}$ over 60 years if $\mathrm{B}$ and $\mathrm{C}$ were foreground stars, whereas, in fact, the separations have remained nearly constant.

The measured changes in separation and position angle are consistent with what would be expected from orbital motion. Star B is at least $760 \mathrm{AU}$ from A. If it is orbiting A, the orbital period of $\mathrm{B}$ is $\gtrsim 13,500 \mathrm{yr}$, and, in 60 years, it would move at most 1.6 in P.A. (depending on the inclination of its orbit). Star C is at least $140 \mathrm{AU}$ from B. If $\mathrm{C}$ is orbiting $\mathrm{B}$, its period is $\gtrsim 2100 \mathrm{yr}$ and in $60 \mathrm{yr}$, it would move at most $10^{\circ}$ in P.A. The relative radial velocities of $B$ and $C$ would be less than $2 \mathrm{~km} \mathrm{~s}^{-1}$. The actual changes in P.A. (see Table 1) and the measured relative radial velocities (see Table 2) are well within these constraints and imply that the stars could be orbiting one another.

Since $\mathrm{A}, \mathrm{B}$, and $\mathrm{C}$ have common proper motions and common radial velocities, they are, with high probability, physical companions. Whether the three stars are actually bound, however, cannot be determined. As noted in Weinberger et al. (1999), if the companion stars are in the plane of the disk, the physical separation of A-B is $990 \mathrm{AU}$ and that of $\mathrm{B}-\mathrm{C}$ is $190 \mathrm{AU}$. Then the ratio of the semimajor axes of the wider to the closer pair would be only $\sim 5.2$. This is not expected to be a stable triple system (Eggleton \& Kiseleva 1995), although such young stars may not yet have had time to become unbound.

The presence of three young stars unassociated with a star-forming cloud begs the question of how they formed. HD 141569 is located in projection near a complex of highlatitude molecular cloud cores, MBM 34-39, associated with the dark clouds L169, L183-4, and L134 (together called L134N). The last of these has $100 \mu$ m emission contours which actually encompass HD 141569 (Sahu et al. 1998). The excess $B-V$ color of HD 141569 A, $0.095 \mathrm{mag}$, implies a reddening $A_{v}=0.3 \mathrm{mag}$, so the star cannot lie deep within the cloud. Sahu et al. (1998) concluded, based on the strength of interstellar absorption lines toward HD $141569 \mathrm{~A}$, that it lies behind part of L134N/MBM 37. However, these clouds have LSR radial velocities of $0.8-3.2$ $\mathrm{km} \mathrm{s}^{-1}$ (Magnani, Blitz, \& Mundy 1985), compared to -20 $\mathrm{km} \mathrm{s}^{-1}$ for HD 141569A, which suggests that they are not comoving with the star. The clouds are also quiescent and compact with internal temperatures of 3-12 K (Snell et al. 1981; Clemens \& Barvainis 1988), which suggests that they are not undergoing protostellar collapse or being heated by nearby stars.

The discovery of other young stars dispersed across the sky has prompted speculation about fast cloud dispersal mechanisms and runaway stars (Feigelson 1996). One way to address the question of star formation in this region is to look for other nearby stars which may have formed at the same epoch as HD 141569. As a first attempt to search for members of such an association, we have queried the Hipparcos catalog using the approximate characteristics of the TW Hya association (Webb et al. 1999), a radius of 19 pc and a proper-motion dispersion of $7 \mathrm{~km} \mathrm{~s}^{-1}$, which, at the distance to HD 141569 , correspond to a circle of radius $10^{\circ}$

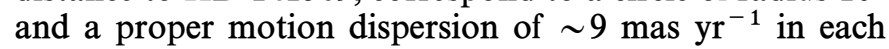
direction. In addition, we require the Hipparcos parallaxes to agree with that of HD 141569A to within their uncertainties. This search produces 14 stars. In comparison, a search of 20 other fields at the same absolute Galactic latitude and a range of Galactic longitudes produces an average of $7 \pm 5$ stars, using the same search parameters. The data suggest an overdensity of stars near HD 141569. We note that, at the distance of 100 pc, the Hipparcos catalog is highly incomplete for stars fainter than $V=9$ mag (later than F).

Two of the 14 stars near HD 141569 are spectral type A and lie below or along the ZAMS (Fig. 6). This location in 
the H-R diagram is populated by young A-type stars (Lowrance et al. 2000) including well-known disk sources such as $\beta$ Pic and HR 4796A, as well as HD 141569 itself.

We have computed the space motion of HD 141569A following Johnson \& Soderblom (1987) and find $U, V$, $W=-3.0,-13.0$, and $-3.0 \mathrm{~km} \mathrm{~s}^{-1}$. This velocity is within twice the dispersion in the space motions of the local star formation associations such as $\eta$ Chameleonitis, TW Hya, and Tucanae (Zuckerman \& Webb 2000). So, the HD 141569 system may have formed as part of a larger episode of star formation near the Sun.

\subsection{Age}

The pre-main-sequence nature of stars $\mathrm{B}$ and $\mathrm{C}$ is confirmed by the presence of lithium in absorption. Since M-type stars should be fully convective, lithium in the spectra indicates that these stars are not yet hot enough in their cores to burn it or have had insufficient time to burn all of it. The equivalent widths of $0.5 \AA$ for $\mathrm{B}$ and $\mathrm{C}$ is very close to the boundary of $0.54 \AA$ set by Martin (1998) for separating weak-line T Tauri stars (WTTS) from post-T Tauri stars. This sets an upper limit on their age of $10 \mathrm{Myr}$ (Martin et al. 1994). The $\mathrm{H} \alpha$ equivalent widths $(<10 \AA)$ also argue for classifying these stars as WTTS, as opposed to classical TTS. The strength of the double-peaked $\mathrm{H} \alpha$ emission is within the distribution of chromospherically active main-sequence M dwarfs (Stauffer \& Hartmann 1986). The central reversal of the line indicates a high level of chromospheric activity, which is generally associated with a variety of factors including youth and rotation. Finally, Lindroos (1985) classified the combined B/C spectrum as "peculiar" because he detected hydrogen and calcium emission lines, and pre-main-sequence stars often have such emission lines.

The $\mathrm{A} / \mathrm{B} / \mathrm{C}$ system falls within the $90 \%$ confidence error circle of a ROSAT sky survey point-source detection. The PSPC count rate of $0.093 \mathrm{~s}^{-1}$ converts to an X-ray luminosity of $8.72 \times 10^{29} \mathrm{ergs} \mathrm{s}^{-1}$, assuming that it comes from stars at 100 pc with T Tauri-like X-ray spectra (Neuhauser et al. 1995). It is likely that the X-ray emission comes from the later-type stars $\mathrm{B}$ and $\mathrm{C}$, rather than from the primary star. We estimate the bolometric luminosities of stars B and $\mathrm{C}$ as $\log \left(L / L_{\odot}\right)=-0.62$ and -0.95 , respectively, from their spectral types and $J$-band magnitudes using bolometric corrections from Hartigan, Strom, \& Strom (1994). This makes their total X-ray to bolometric luminosity ratio $\log \left(L_{\mathrm{X}} / L_{\mathrm{bol}}\right)=-3.2$. For pre-main-sequence stars, the ratio of X-ray to bolometric luminosity increases with stellar age, and the ratio for stars $\mathrm{B} / \mathrm{C}$ is typical for that of stars in clusters of age less than $20 \mathrm{Myr}$ (Kastner et al. 1997).

Finally, we estimate the age of stars B and C on the basis of their location on theoretical pre-main-sequence evolutionary tracks. Here, a major source of uncertainty is the not well-understood effective temperature scale for $\mathbf{M}$ dwarfs (Allard et al. 1997). Following the spectral-type to temperature calibration of Luhman \& Rieke (1998), we can assign an effective temperature for B (M2 V) and C (M4 V) of $3500 \pm 85 \mathrm{~K}$ and $3200 \pm 85 \mathrm{~K}$, respectively, where the uncertainties correspond to one-half spectral subclass. We note that the temperature scale of Kirkpatrick et al. (1993) gives a temperature $175 \mathrm{~K}$ hotter for M4 V.

Shown in Figure 7 are tracks by Baraffe et al. (1998), with the effective temperatures and absolute $J$-band magnitudes of stars B and C plotted. The plotted magnitudes assume that stars B and C are at the same distance as A, $100 \mathrm{pc}$, and

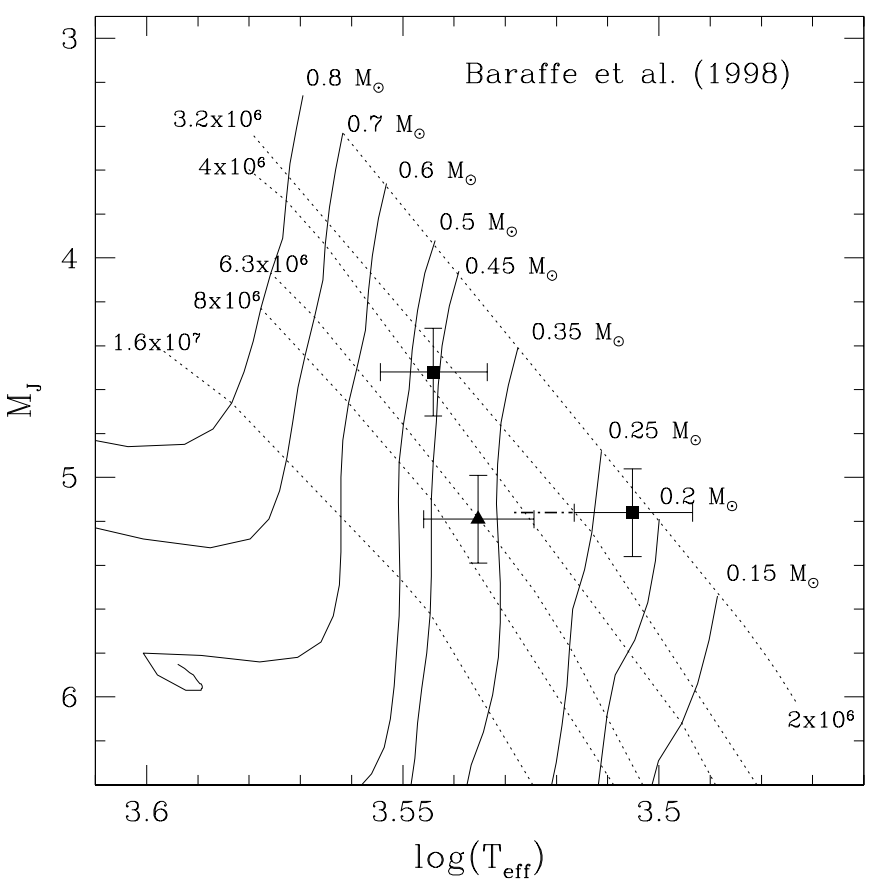

Fig. 7.-Absolute $J$-band magnitudes and temperatures of stars B (brighter) and C (fainter) plotted (filled squares) on pre-main-sequence tracks by Baraffe et al. (1998). The same quantities are also plotted for HR 4796B from Jura et al. 1993 (filled triangle). The effective temperatures and uncertainties thereon are from Luhman \& Rieke (1998), but the dashdotted line on the effective temperature of $\mathrm{C}$ shows the range of M4 $\mathrm{V}$ temperatures from other authors. The age of HD $141569 \mathrm{~B} / \mathrm{C}$ appears to be 2-8 Myr, about a factor of 2 younger than HR 4796B.

the uncertainties in the magnitudes correspond to the uncertainty in the Hipparcos parallax to A. Within the uncertainties, stars B and C appear to be the same age of 2-8 Myr. Star A is indistinguishable from the ZAMS and is not shown on the figure. The tracks indicate masses for stars B and C of 0.5 and $0.25 M_{\odot}$, respectively. Tracks by other authors give the same basic result. For comparison, Figure 7 also shows HR 4796B, with an effective temperature of an M2.5 star from Luhman \& Rieke (1998). This companion to HR 4796A, another star with a well-studied circumstellar disk, was assigned an age of $8 \pm 2 \mathrm{Myr}$ by Stauffer et al. (1995) using tracks from D'Antona \& Mazzitelli (1994). The age given by the Baraffe et al. (1998) tracks is consistent with that determination and shows that HD $141569 \mathrm{~B} / \mathrm{C}$ are about a factor of 2 younger than HR 4796B.

Based on all of the above arguments, we estimate an age for the HD 141569 system of $5 \pm 3 \mathrm{Myr}$.

\subsection{Dynamics}

The disk around HD 141569A has two features which suggest dynamical sculpting (Weinberger et al. 1999). First, the density of scatterers is as high at $360 \mathrm{AU}$ as $200 \mathrm{AU}$ from the star. If the companions are out of the plane of the disk, they could excite significant vertical velocities in the disk dust.

Second, there is a dip in surface brightness, or a "gap" in the disk at a radius of $250 \mathrm{AU}$ with a width of $60 \mathrm{AU}$. No point source is seen in the gap to a limit of $F 110 \mathrm{~W}=20.3$ mag. The gap is as circular as the disk and must be cleared continually to remove particles drawn through it by radiation pressure and Poynting-Robertson drag. If the com- 
panion stars orbit each other and the primary and are in the plane of the disk, their center of mass is $1053 \mathrm{AU}$ from the primary. The 2:1-9:1 Lindblad resonances between the orbital period of the companion center of mass and orbiting particles in the disk are shown with the dashed ellipses on Figure 1. There is no obvious agreement between the resonances and the structure observed in the disk, even if the companions are assumed to be somewhat out of the plane, and so the resonances shifted. The resonances closest to the gap lie at $243 \mathrm{AU}(9: 1)$ and $263 \mathrm{AU}(8: 1)$ from the star. It is not clear, however, why only these high-order resonances, and not any of the others, would mold the disk. If the companions were in very eccentric orbits and currently near apastron, the locations of the resonances might change dramatically with time. However, the circularity of the gap implies a stable dynamical influence over long time periods.

\section{CONCLUSIONS}

On the basis of common proper motion, common radial velocity, and the low probability of a chance superposition of three young stars away from any known star-forming cloud, we conclude that HD $141569 \mathrm{~A} / \mathrm{B} / \mathrm{C}$ form a physical association which may or may not be bound. The age of the stars as determined from spectroscopic features, X-ray emission, and placement on pre-main-sequence tracks is $5 \mathrm{Myr}$. At least two other stars, HD 141693 and HD 140574, may be members of this common proper motion group.

HD 141569 has a now well-studied disk with grains present within 25-500 AU of the star. The inferred size of the grains, less than $5 \mu \mathrm{m}$ (Fisher et al. 2000), means that radiation pressure blows them away in at least an order of magnitude less time than our derived stellar age. Thus, they must be continuously regenerated, probably through collisions of larger bodies. The timescales for the formation of planetesimal cores is $10^{4}-10^{5} \mathrm{yr}$ (Wetherill 1980), which is easily consistent with the age of the system. From measurements of CO around HD 141569, the remnant mass of $\mathrm{H}_{2}$ is estimated as 20-460 $M_{\text {Earth }}$ (Zuckerman, Forveille, \& Kastner 1995), so any gas giants present must have formed very quickly.

The morphology of the disk, including a gap at $250 \mathrm{AU}$, indicates the presence of dynamical sculpting. Resonant interactions between the companions and the disk do not appear to account for the structure, and it would be hard, given current models of planet formation, to generate Jupiter-sized bodies in a disk of such young age at distances so far from the central star (Boss 1998). The cause of the structure in the disk thus remains unknown.

We thank G. Neugebauer for donating 200 inch Telescope time to this project. We thank B. Schaefer and R. Quick for making the LRIS observations at the W. M. Keck Observatory, which is operated as a scientific partnership between the California Institute of Technology, the University of California, and the National Aeronautics and Space Administration, and made possible by the generous financial support of the W. M. Keck Foundation. This work is supported in part by NASA grant NAG 5-3042, and based on observations with the NASA/ESA Hubble Space Telescope, obtained at the Space Telescope Science Institute, which is operated by the Association of Universities for Research in Astronomy, Inc., under NASA contract NAS 5-26555. We also thank M. Jura for many helpful discussions.
Allard, F., Hauschildt, P. H., Alexander, D. R., \& Starrfield, S. 1997, ARA\& $\&, 35,137$

Andrillat, Y., Jaschek, M., \& Jaschek, C. 1990, A\&A, 233, 474

Augereau, J. C., Lagrange, A. M., Mouillet, D., \& Menard, F. 1999, A\&A, 350,51

Baraffe, I., Chabrier, G., Allard, F., \& Hauschildt, P. H. 1998, A\&A, 337, 403

Barrado y Navascués, D., Stauffer J. R., Hartmann, L., \& Balachandran, S. 1997, ApJ, 475, 313

Barrado y Navascués, D., Stauffer, J. R., Song, I., \& Caillault, J-P. 1999, ApJ, 520, 123

Boss, A. P. 1998, ApJ, 503, 923

Clemens, D. P., \& Barvainis, R. 1988, ApJS, 68, 257

D'Antona, F., \& Mazzitelli, I. 1994, ApJS, 90, 467

Dunkin, S. K., Barlow, M. J., \& Ryan, S. G. 1997, MNRAS, 286, 604

Eggleton, P., \& Kiseleva, L. 1995, ApJ, 455, 640

Feigelson, E. D. 1996, ApJ, 468, 306

Fisher, R. S., Telesco, C. M., Piña, R. K., Knacke, R. F., \& Wyatt, M. C. 2000, ApJ, 532, 141

Frisch, P. C. 1987, ApJS, 65, 313

Gahm, G. F., Ahlin, P., \& Lindroos, K. P. 1983, A\&AS, 51, 143

Habing, H. J., Dominik, C., Jourdain De Muizon, M., Kessler, M. F., Laureijs, R. J., Leech, K., Metcalfe, L., Salama, A., Siebenmorgen, R., \& Trams, N. 1999, Nature, 401, 456

Hartigan, P., Strom, K. M., \& Strom, S. E. 1994, ApJ, 427, 961

Herbig, G. H. 1960, ApJS, 4, 337

Hillenbrand, L. A., Strom, S. E., Vrba, F. J., \& Keene, J. 1992, ApJ, 397, 613

Johnson, D. R. H., \& Soderblom, D. R. 1987, AJ, 93, 864

Jura, M., Malkan, M., White, R., Telesco, C., Pina, R., \& Fisher, R. S. 1998, ApJ, 505, 897

Kastner, J. H., Zuckerman, B., Weintraub, D. A., \& Forveille, T. 1997, Science, 277, 67

Kirkpatrick, J. D., Henry, T. J., \& McCarthy, D.W., Jr. 1991, ApJS, 77, 417

Kirkpatrick, J. D., Kelly, D. M., Rieke, G. H., Liebert, J., Allard, F., \& Wehrse, R. 1993, ApJ, 402, 643

Lindroos, K. P. 1985, A\&AS, 60, 183
Lowrance, P. J., et al. 2000, ApJ, 541, 390

Luhman, K. L., \& Rieke, G. H. 1998, ApJ, 497, 354

Magnani, L., Blitz, L., \& Mundy, L. 1985, ApJ, 295, 402

Malfait, K., Bogaert, E., \& Waelkens, C. 1998, A\&A, 331, 223

Martin, E. L. 1998, AJ, 115, 351

Martin, E. L., Rebolo, R., Magazzu, A., \& Pavlenko, Y. V. 1994, A\&A, 282, 503

Neuhauser, R., Sterzik, M. F., Schmitt, J. H. M. M., Wichmann, R., \& Krautter, J. 1995, A\&A 297, 391

Oke, J. B., Cohen, J. G., Carr, M., Cromer, J., Dingizian, A., Harris, F. H., Labrecque, S., Lucinio, R., \& Schaal, W. 1995, PASP, 107, 375

Pirzkal, N., Spillar, E. J., \& Dyck, H. M. 1997, ApJ, 481, 392

Rossiter, R. A. 1943, Publ. Michigan Obs., 8, 133

. 1955, Publ. Michigan Obs., 11, 1

Sahu, M. S., Blades, J. C., He, L., Hartmann, D., Barlow, M. J., \& Crawford, I. A. 1998, ApJ, 504, 522

Silverstone, M. D. 2000, Ph.D. thesis, UCLA

Silverstone, M. D., et al. 1998, BAAS, 30, 1363

Snell, R. L. 1981, ApJS, 45, 121

Spangler, C., Silverstone, M. D., Becklin, E. E., Hare, J., Zuckerman, B., Sargent, A., \& Goldreich, P. 1999, in The Universe as Seen by ISO, ed. P. Cox \& M. F. Kessler (ESA SP-427; Paris: ESA), 405

Stauffer, J. R., \& Hartmann, L. W. 1986, ApJS, 61, 531

Stauffer, J. R., Hartmann, L. W., \& Barrado y Navascués, D. 1995, ApJ, 454, 910

The, P. S., de Winter, D., \& Perez, M. R. 1994, A\&AS, 104, 315

Vogt, S. S., et al. 1994, Proc. SPIE, 2198, 362

Webb, R. A., Zuckerman, B., Platais, I., Patience, J., White, R. J., Schwartz, M. J., \& McCarthy, C. 1999, ApJ, 512, L63

Weinberger, A. J., Becklin, E. E., Schneider, G., Smith, B. A., Lowrance, P. J., Silverstone, M. D., Zuckerman, B., \& Terrile, R. J. 1999, ApJ, 535, L53 Wetherill, G. W. 1980, ARA\&A, 18, 77

Zuckerman, B. 1995, in Circumstellar Dust Disks and Planet Formation, ed. R. Ferlet, \& A. Vidal-Madjar (Edition Frontières: Paris), 131

Zuckerman, B., Forveille, T., \& Kastner, J. H. 1995, Nature, 373, 494

Zuckerman, B., \& Webb, R. A. 2000, ApJ, 535, 959 